\title{
Imaging Proton Transport in Giant Vesicles through Cyclic Peptide- Polymer Conjugate Nanotube Transmembrane Ion Channels
}

Jason G. Binfield, ${ }^{\mathrm{a}}$ Johannes C. Brendel, ${ }^{\mathrm{a} \neq}$ Neil R. Cameron*,,c Ahmed M. Eissa ${ }^{\mathrm{b}, \mathrm{c}, \mathrm{d} *}$ and Sébastien Perrier ${ }^{\mathrm{a}, \mathrm{e}, \mathrm{f} *}$

${ }^{a}$ Department of Chemistry, The University of Warwick, Coventry CV4 7AL, U.K.;

${ }^{b}$ School of Engineering, The University of Warwick, Coventry CV4 7AL, U.K.;

${ }^{c}$ Department of Materials Science and Engineering, Monash University, 22 Alliance Lane, Clayton, 3800, Victoria, Australia;

${ }^{d}$ Department of Polymers, Chemical Industries Research Division, National Research Centre (NRC), 33 El-Bohouth Street, Dokki, Giza, 12622, Cairo, Egypt;

${ }^{e}$ Warwick Medical School, The University of Warwick, Coventry CV4 7AL, U.K.;

${ }^{f}$ Faculty of Pharmacy and Pharmaceutical Sciences, Monash University, VIC 3052, Australia;

‡ Current address: Jena Center for Soft Matter (JCSM), Friedrich-Schiller-University, Philosophenweg 7, 7743 Jena, Germany.

Corresponding Authors:

* Email: $\underline{\text { A.M.Eissa@warwick.ac.uk }}$

*Email: S.Perrier@warwick.ac.uk

Since their discovery in 1993, interest in various aspects of cyclic peptides (CPs) has expanded rapidly. Of particular note is their potential to form artificial ion channels in lipid membranes, an attractive characteristic in supramolecular chemistry and biological 
research. The design and synthesis of cyclic peptide-polymer conjugates (CPPCs) that can self-assemble within lipid bilayers into nanotubes, mimicking naturally occurring membrane channels and pores, has been reported. However, methods that allow direct detection of the transport process with high levels of certainty are still lacking. This work focuses on the development of a simple but reliable approach to verify and quantify proton transport across a bilayer membrane. Giant unilamellar vesicles (GUVs) are created via the electroformation method and CPPCs are incorporated in GUV membranes at varying concentrations $(\mathbf{0 - 1 0 \%})$. Confocal fluorescence microscopy is used to demonstrate full inclusion of fluorescein-labelled CPPCs in the GUV membranes. The pH sensitive dye, carboxyfluorescein is encapsulated within the water pool of the GUVs and used as an indicator of proton transport. This assay is versatile and can be exploited on other existing proton transporter systems, providing a consistent tool to compare their performances. It should also aid the development of novel antineoplastics and drug delivery systems.

\section{Introduction}

Ion transport forms an essential part of the cell life-cycle, and is responsible for many functions of the cell. Transport across membranes often results from an uneven distribution of ions in cells and tissue. For example, the intracellular concentration of potassium $\left(\mathrm{K}^{+}\right)$is higher than its extracellular concentration, while sodium $\left(\mathrm{Na}^{+}\right)$and chloride $\left(\mathrm{Cl}^{-}\right)$ions are at higher concentrations in the extracellular region than in the intracellular regions. The ion concentration gradients are regulated by the gating action of ion channel proteins, known as ionophores that are buried in the relatively impermeable bilayer membranes. ${ }^{[1]}$ Maintenance of precise ion gradients across the cell membrane is critical for cells to avoid osmolysis, stimulate 
energy that drives metabolic processes and control electrical signalling in nerves and muscles. ${ }^{[2]}$

The development of artificial ion channels and nanopores that can mimic the functions of the natural transmembrane proteins is of great importance, not only to understand the fundamental structure-function relationship of complex channel proteins at the molecular level, but also for (bio)technological applications, such as sensing, ${ }^{[3]}$ separation $^{[4]}$ and diagnosing and/or managing diseases. ${ }^{[5]}$ To date, several amphiphilic compounds that insert into the bilayer and form pores either on their own or by self-assembly have been proposed as artificial ion channels. ${ }^{[6]}$ Although, the majority of synthetic ionophores are based on macrocycles such as crown ethers, cryptands, calixarenes and cyclodextrins, some other examples exist such as phenols, which exhibit ionophoric properties. ${ }^{[7]}$ In order to generate artificial ion channels, a number of parameters need to be optimised such as pore size and ion selectivity/inhibition of the channels; furthermore, the facile cost-effective synthesis and biocompatibility of the synthetic ionophores are other important factors to be considered. ${ }^{[8,9]}$

Recently, several studies highlighted the potential of self-assembled cyclic peptide nanotubes to create synthetic transmembrane channels. ${ }^{[10,11]}$ These nanotubular materials are based on cyclic peptide (CP)-polymer conjugates consisting of two polymer chains (either "Janus" or hybrid shells) directly attached to a single $\mathrm{CP}$ core. ${ }^{[12]}$ They are able to form micropores in the phospholipid bilayers of large unilamellar vesicles (LUVs) offering a functional utility and subnanometer dimensions similar to naturally occurring transmembrane protein channels. Synthetic polymers are attractive species to attach to cyclic peptides due to their tunability in length, functionality and hydrophobicity as well as their ability to control the strength of interactions, providing increased solubility of the cyclic peptide nanotubes. ${ }^{[13-15]}$ These systems can also be temperature responsive, if based upon CP-poly( $N$-isopropylacrylamide) conjugates, opening up opportunities for developing novel systems for hyperthermic drug delivery 
applications. ${ }^{[16]}$ Ion transport studies in LUVs are commonly performed using a fluorescence technique involving an ion-sensitive fluorophore to monitor the transport into and out of the vesicles. This method is easy to implement and provides semi-quantitative transport measures, but does not provide a comprehensive description of the transport process. LUVs, being around 100-200 $\mathrm{nm}$ in diameter, are at least 10-fold smaller than most cells, limiting their relevance as cell models and presenting challenges in their visualisation Furthermore, typical reports of successful transport into or out of vesicles refer to observations in a change in fluorescence, but in principle this change could be associated with vesicle bursting rather than transport across the membrane. Hence, there is a need for a complementary technique that would allow unambiguous observation of the transport process, prior to implementation of potential agents in biological systems.

Herein, we report a new approach that demonstrates unambiguously the formation of transbilayer channels using CP-poly(butyl acrylate) conjugates and allows direct observation of the transport process. In this approach, we employ cell-sized giant unilamellar vesicles (GUVs) that can be readily observed by microscopy. Cyclic peptide polymer conjugates are incorporated within the bilayer membrane of GUVs made from 1,2-dioleoyl-sn-glycero-3phosphocholine (DOPC) lipid, under electroformation conditions. Confocal fluorescence microscopy is used to visualise the transmembrane transport process, which is directly imaged by quenching of the $\mathrm{pH}$-sensitive dye carboxyfluorescein. We also confirm the complete integration of the $\mathrm{CP}$ channels in the GUV membranes by utilizing fluorescein-labelled cyclic peptide-polymer conjugates.

\section{Results and Discussion}


The cyclic peptide-polymer conjugate nanotubes employed in this study are based on cyclic peptides containing eight alternating $\alpha-\mathrm{D}$, L-amino acid residues that have been previously described in the literature. ${ }^{[17]}$ The linear peptide (d-Leu-Lys-d-Leu-Trp) 2 was first produced via solid-phase peptide synthesis. Following a progressive protection/deprotection protocol, the amino acids were added one by one to the resin using O-(6-chlorobenzotriazol-1-yl)- $N, N, N^{\prime}, N^{\prime}-$ tetramethyluronium hexafluorophosphate (HCTU) as a coupling reagent. Lysine was used in the peptidic structure to provide two coupling sites on opposite ends of the cyclic peptide, and incorporation of tryptophan residues increases solubility of the peptide. Finally the leucine residue is used to further stabilise the structure by hydrophobic interactions. ${ }^{[18]}$ Cyclisation reaction of the linear peptide was then carried out using 4-(4,6-dimethoxy-1,3,5-triazin-2-yl)4-methylmorpholinium tetrafluoroborate $\left(\mathrm{DMTMM} \mathrm{BF}_{4}\right)$ as an efficient intramolecular peptide coupling reagent. ${ }^{[19]}$ Once cyclized, the Boc groups were removed under typical deprotection conditions to offer cyclo(d-Leu-Lys-d-Leu-Trp) $)_{2}$, Scheme 1 . The design of this cyclic peptide allows the subsequent conjugation of functional polymeric moieties through the lysine $\mathrm{NH}_{2}$-groups, providing a cyclic peptide structure with two opposite polymeric arms.

\section{Scheme 1.}

We synthesized carboxylic acid end-capped poly(n-butyl acrylate) (p(BA)) via RAFT polymerization using dimethyl 2,2'-azobis(2-methylpropionate) (V-601) as initiator and 2(((butylsulfanyl)carbothioyl)sulfanyl)propanoic acid (PABTC) as a RAFT agent, ESI. The RAFT polymerization reactions of BA were terminated before full conversion to prevent side reactions of polymer chains, producing polymers with a high degree of control as demonstrated by their low dispersities (ESI). We then conjugated the prepared $\mathrm{p}(\mathrm{BA})_{33}$ to the cyclic peptide 
using either 2-(1H-benzo-triazol-1-yl)-1,1,3,3-tetramethyluronoium hexafluorophosphate (HBTU) or benzotriazol-1-yl-oxytripyrrolidinophosphonium hexafluorophosphate (PyBOP) as a coupling agent, ESI. We found that the coupling reaction employing PyBOP is more efficient compared to HBTU, as revealed from GPC traces of reactions monitored at $30 \mathrm{~min}, 120 \mathrm{~min}$, and $24 \mathrm{~h}$ in DMF, using $N, N$-dimethyldiisopropylamine (DIPEA) in excess (ESI). DIPEA is needed in the coupling reactions to preactivate the carboxylic acid and to increase reactivity to prevent side reactions such as guanidine formation. ${ }^{[20]}$ After 30 min., the PyBOP-mediated coupling reaction has significantly less $\mathrm{p}(\mathrm{BA})$, compared to the HBTU-mediated reaction, and surpasses the total coupling of the HBTU-mediated reaction after 24 hours. We have also copolymerized fluorescein- $O$-acrylate (FA) with $\mathrm{p}(\mathrm{BA})$ to provide a fluorescent CPPC, ESI. The copolymerization of BA and FA was carried out under RAFT conditions and the following CP coupling reaction was performed using a similar method to that described above (ESI).

Due to their adequate sizes for imaging, giant unilamellar vesicles (GUVs) have received much attention for studies requiring cell mimics. ${ }^{[21]}$ GUV membranes can also be modified to enhance cell mimicry, for example by embedding artificial channels in the membrane. ${ }^{[22]}$ One successful method to produce GUVs with lower defect density is electroformation whereby lipids are placed on a conductive glass followed by applying an AC electric field. ${ }^{[23,24]}$ Electroformation is reported to allow faster and better controlled formation of GUVs comparedwith spontaneous processes (film rehydration). ${ }^{[25]}$ Recent developments in the electroformation method have shown its proficiency to form GUVs from zwitterionic lipid species $^{[26]}$ as well as its capacity to utilize a variety of electrolyte-containing buffers. ${ }^{[21,27]}$

GUVs were formed from 1,2-dioleoyl-sn-glycero-3-phosphocholine (DOPC) lipid using a custom-made electroformation apparatus (ESI). ${ }^{[1]}$ A DOPC film was solution-cast in the presence of Nile Red on the surfaces of both indium tin oxide-coated glass slide electrodes. Application of an AC voltage between the two electrodes during the swelling of the lipid in 
water resulted in the formation of GUVs of 1-20 $\mu \mathrm{m}$ in diameter as revealed by confocal fluorescence microscopy, Figure 1A and B. We then introduced the BA CPPCs into the DOPC films at different mass ratios $(2,5,10 \%)$. Interestingly, size distribution studies of GUVs revealed a trend that is a decrease in size as CPPC content increases, Figure 1C. While all samples are predominantly occupied by GUVs of $2 \mu \mathrm{m}$, the distributions gradually transition from more GUVs of $3 \mu \mathrm{m}$ to GUVs of $1 \mu \mathrm{m}$ as CPPC content increases, Figure 1D. It is noteworthy that although the sample containing GUVs with 10\% CPPC does not clearly follow this trend, it still displays a shift in distribution towards smaller GUVs compared to the control (no CPPC). This preliminary observation already suggests that the CPPC affect the structure of GUVs, possibly by interacting with their lipid bilayer.

\section{Figure 1.}

In order to confirm the integration of CPPCs in the bilayer membrane of GUVs, we added the BA/FA CPPC at 5\% mass into the DOPC during film formation prior to the electroformation process. Nile Red was added to the DOPC/CPPC solution used in the film formation. Confocal fluorescence microscopy was used to visualize the electroformed Nile Red-labelled GUVs (red channel, $514 \mathrm{~nm}$ ), Figure 2. As hypothesized, upon excitation with a $488 \mathrm{~nm}$ laser, the GUVs showed significant integration of fluorescein-labelled CPPC throughout the GUV membranes (green channel), Figure 2D-F. Upon further magnification, we can clearly observe bright green specks along the top-left perimeter of the GUV which are not present in the red channel image, Figure $2 \mathrm{~F}$. We presume that these green specks represent areas of high concentrations of fluorescein-labelled CPPC. These differing areas of intensity between the two images, suggest that integration of the cyclic peptides has occurred. A control experiment was performed where 
no fluorescein-labelled CPPC was added to the Nile Red-labelled GUV membranes. In this case, no GUVs were observed under the green channel.

\section{Figure 2.}

In order to investigate the ability of the CPPCs to form channels (pores) in the bilayer membrane, carboxyfluorescein-encapsulated GUVs containing various levels of CPPC content were produced. Carboxyfluorescein is a $\mathrm{pH}$-responsive fluorescent dye that has a strong emission at neutral $\mathrm{pH}$ but a weak emission in acidic $\mathrm{pH}$. We hypothesized that the formation of CPPC channels will allow diffusion of protons across the GUV membrane. We studied the changes in fluorescence intensity of carboxyfluorescein-encapsulated GUVs upon addition of hydrochloric acid. Aliquots of $6 \mu \mathrm{L}$ of $2 \mathrm{M}$ hydrochloric acid were added to the visualization chamber containing the GUVs in phosphate buffered aqueous solution and confocal images were taken at regular time intervals for $20 \mathrm{~min}$. The confocal microscope was set to an equal gain level for each time series, and the mean gray value of the carboxyfluorescein channel was measured both inside and outside of the GUVs, so as to subtract background fluorescence. Beyond 25 min of acid addition, the fluorescence measurements become more challenging as a result of GUV aggregation over time and/or GUV degradation. As expected, an increased CPPC content increased the speed of quenching, most probably due to increasing the number of proton channels. Figure 3 clearly shows the difference in fluorescence between GUVs containing 0\% CPPC and GUVs containing 2, 5 and 10\% CPPC. The sample containing GUVs with $0 \% \mathrm{CPPC}$ had the highest fluorescence within the GUVs, which was maintained at all measurement times. The rates of diffusion appear to be greater in the CPPC infused GUVs, 
showing a dramatic decrease in the sample containing 10\% CPPC infused GUVs until it reaches a plateau after only $10 \mathrm{~min}$.

\section{Figure 3.}

\section{Conclusion}

In conclusion, we have developed a protocol for the direct observation of proton transfer across a lipid bilayer promoted by the self-assembly of a CPPC. To the best of our knowledge, this is the first report in which cyclic peptides are incorporated in a GUV membrane and the proton transport process has been visualized and quantified. Beyond opening the path to biological application of the CPPC systems, this report also proposes a methodology that could in principle be generalized to characterize other ion transport processes.

\section{Supporting Information}

Experimental details including materials, instruments and analysis, methods and proof of structures.

\section{Acknowledgements}

We thank the Monash-Warwick Alliance (A.M.E.; N.R.C.; S.P.), the Royal Society Wolfson Merit Award (WM130055; S.P.) and the European Research Council (TUSUPO 647106; S.P.) for financial support. J.C.B. thanks the German Science Foundation (DFG) for granting a full postdoctoral fellowship (BR 4905/1-1). 


\section{Conflict of Interest}

The authors declare no conflict of interest.

\section{Keywords}

cyclic peptide-polymer conjugates, supramolecular chemistry, nanotubes, giant unilamellar vesicles, proton transport, artificial ion channels, biological membranes.

\section{References}

[1] A. P. Davis, D. N. Sheppard, B. D. Smith, Chem. Soc. Rev. 2007, 36, 348.

[2] Y. F. Li, S. F. Wang, G. W. He, H. Wu, F. S. Pan, Z. Y. Jiang, Chem. Soc. Rev. 2015, $44,103$.

[3] S. Litvinchuk, H. Tanaka, T. Miyatake, D. Pasini, T. Tanaka, G. Bollot, J. Mareda, S. Matile, Nat. Mater. 2007, 6, 576.

[4] D. L. Gin, R. D. Noble, Science 2011, 332, 674.

[5] J. J. Kasianowicz, Chem. Rev. 2012, 112, 6215.

[6] G. W. Gokel, S. Negin, Acc. Chem. Res. 2013, 46, 2824.

[7] N. Sakai, J. Mareda, S. Matile, Molecular Biosystems 2007, 3, 658.

[8] A. L. Sisson, M. R. Shah, S. Bhosale, S. Matile, Chem. Soc. Rev. 2006, 35, 1269.

[9] R. N. Das, Y. P. Kumar, O. M. Schutte, C. Steinem, J. Dash, J. Am. Chem. Soc. 2015, 137,34 .

[10] D. Wang, L. Guo, J. Zhang, L. R. Jones, Z. Chen, C. Pritchard, R. W. Roeske, J. Pept. Res. 2001, 57, 301. 
[11] M. R. Ghadiri, J. R. Granja, L. K. Buehler, Nature 1994, 369, 301.

[12] M. Danial, C. M. N. Tran, P. G. Young, S. Perrier, K. A. Jolliffe, Nat. Commun. 2013, 4,13 .

[13] M. Danial, S. Perrier, K. A. Jolliffe, Organic \& Biomolecular Chemistry 2015, 13, 2464.

[14] J. Couet, J. D. Jeyaprakash, S. Samuel, A. Kopyshev, S. Santer, M. Biesalski, Angew. Chem.-Int. Edit. 2005, 44, 3297.

[15] R. M. Broyer, G. N. Grover, H. D. Maynard, Chem. Commun. 2011, 47, 2212.

[16] M. Danial, C. M. N. Tran, K. A. Jolliffe, S. Perrier, J. Am. Chem. Soc. 2014, 136, 8018.

[17] M. R. Ghadiri, K. Kobayashi, J. R. Granja, R. K. Chadha, D. E. McRee, Angew. Chem.Int. Edit. Engl. 1995, 34, 93.

[18] J. Montenegro, M. R. Ghadiri, J. R. Granja, Acc. Chem. Res. 2013, 46, 2955.

[19] Z. J. Kaminski, B. Kolesinska, J. Kolesinska, G. Sabatino, M. Chelli, P. Rovero, M. Blaszczyk, M. L. Glowka, A. M. Papini, J. Am. Chem. Soc. 2005, 127, 16912.

[20] A. El-Faham, F. Albericio, Chem. Rev. 2011, 111, 6557.

[21] A. Kubilis, A. Abdulkarim, A. M. Eissa, N. R. Cameron, Sci Rep 2016, 6, 8.

[22] H. Valkenier, N. L. Mora, A. Kros, A. P. Davis, Angew. Chem.-Int. Edit. 2015, 54, 2137.

[23] M. I. Angelova, D. S. Dimitrov, Faraday Discuss. 1986, 81, 303.

[24] T. J. Politano, V. E. Froude, B. X. Jing, Y. X. Zhu, Colloid Surf. B-Biointerfaces 2010, $79,75$.

[25] P. Walde, K. Cosentino, H. Engel, P. Stano, Chembiochem 2010, 11, 848.

[26] N. Rodriguez, F. Pincet, S. Cribier, Colloid Surf. B-Biointerfaces 2005, 42, 125.

[27] T. Pott, H. Bouvrais, P. Meleard, Chem. Phys. Lipids 2008, 154, 115. 


\section{Captions to figures/schemes}

Scheme 1. A) Reversible addition - fragmentation chain - transfer (RAFT) polymerization of n-butyl acrylate (BA), B) peptide coupling C) copolymerization of BA and fluorescein Oacrylate (FA)

Figure 1. Preparation of GUVs from DOPC films containing CPPC. A, B) confocal fluorescence micrographs of GUVs stained with Nile Red, a membrane marker; C) size distribution frequency graph obtained from confocal Z-stack images. Despite fluctuations, there is no correlation between \% CP in the GUV film and GUV sample density, D) normalized size distribution graph obtained from confocal Z-stack images. As \% CP film increases, there is a shift in size distribution to higher percentages of smaller vesicles

Figure 2. Confocal microscopy images of GUVs showing fluorescein labelled CPPCs are incorporated into GUV membranes: A-C) red channel (Nile Red $514 \mathrm{~nm}$ ); D-F) green channel (Fluorescein $488 \mathrm{~nm}$ ). Image F shows clusters of CPPCs in the top left corner (green specks). Scale bar $=20 \mu \mathrm{m}$.

Figure 3. Evaluation of dye (carboxyfluorescein) quenching in GUVs of variable CPPC content 


\section{Table of Contents}

A simple strategy to visualize and quantify proton transport across lipid bilayers through self-assembled cyclic peptide-polymer conjugate (CPPC) nanotubes incorporated in an electroformed giant unilamellar vesicle (GUV) membrane is presented. Confocal fluorescence microscopy shows comprehensive integration of fluorescein-labelled CPPCs in the GUV membranes. Formation of ion channels is confirmed by $\mathrm{pH}$-sensitive dye quenching experiments. 


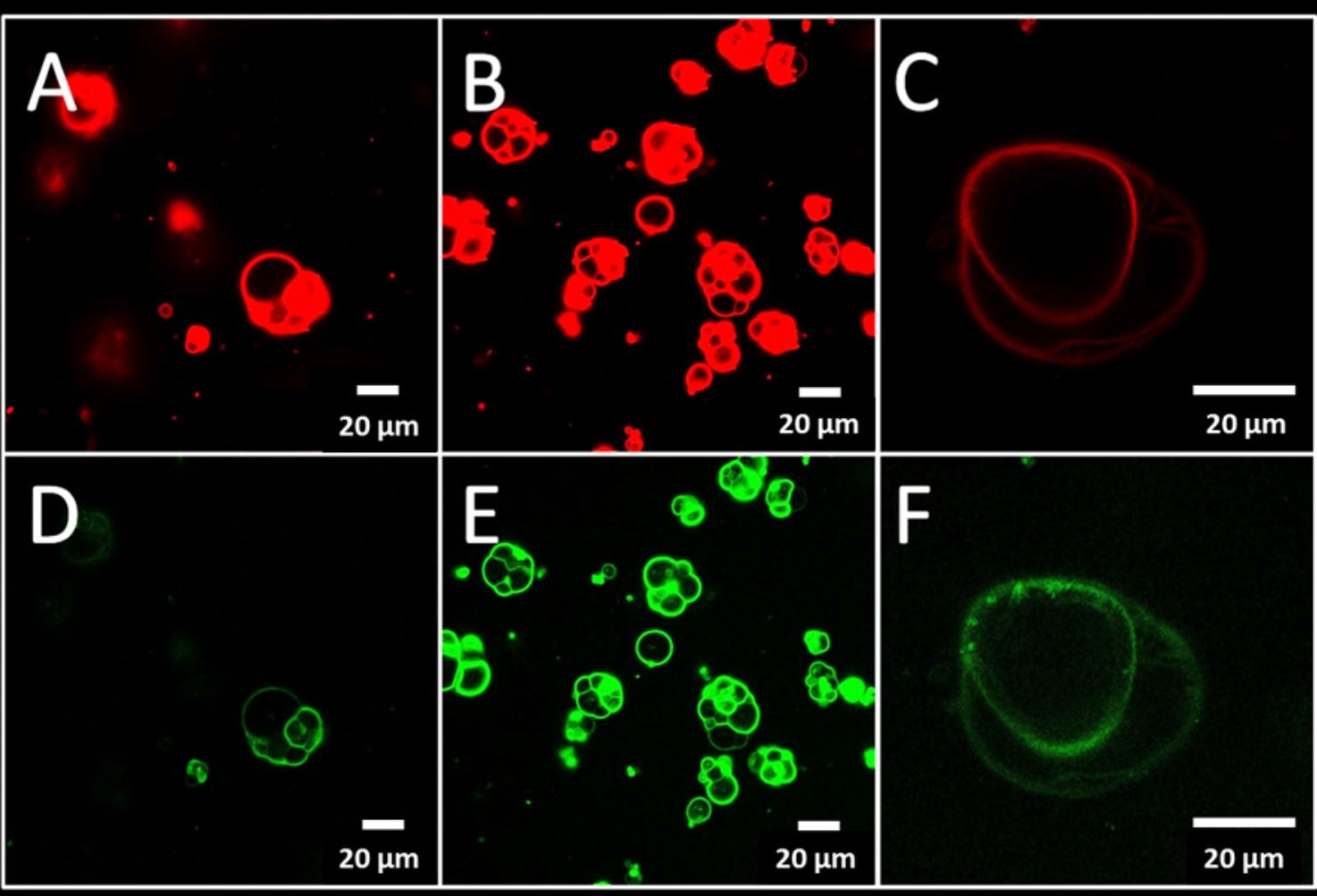




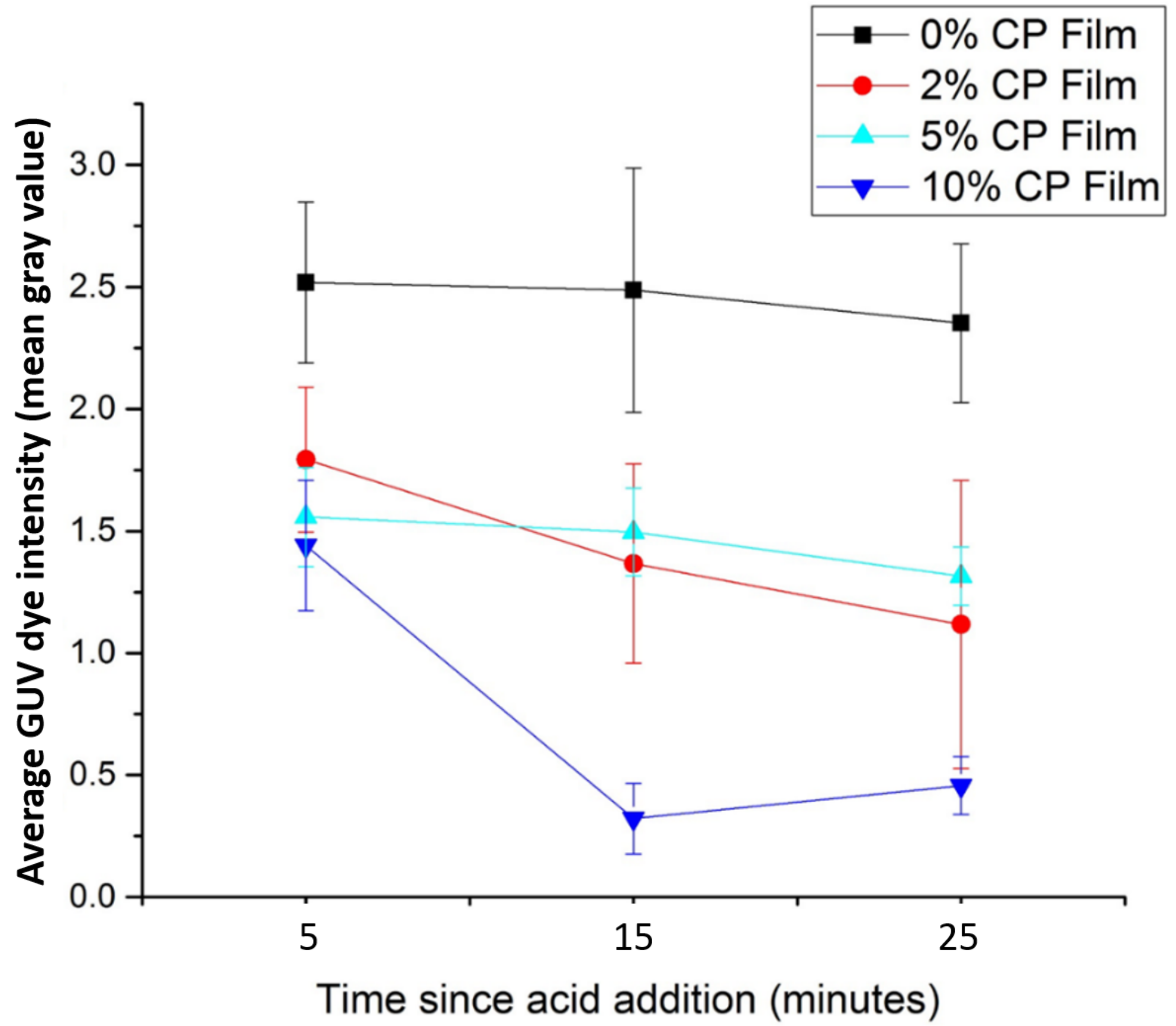


